\title{
Energy saving strategy for the development of icephobic coatings and surfaces
}

\author{
Yan Zheng, Jie Wang, Junpeng Liu, Kwing-So Choi, Xianghui Hou*
}

Faculty of Engineering, The University of Nottingham, Nottingham, NG7 2RD, UK

Corresponding author: xianghui.hou@ nottingham.ac.uk, Tel: +44-1159513920

\begin{abstract}
Aircraft are frequently exposed to cold environments and ice accumulation on aircraft surface may lead to catastrophic accidents. An effective solution of ice protection is a critical requirement in the aerospace industry. For the research and development of icephobic coatings, the current coating design target mainly focuses on lowering the ice adhesion strength between the ice and the surface. However, as a passive ice protection approach, the use of icephobic coating often has to be combined with an active ice protection solution (e.g. electro-thermal heating, hot air bleeding, and vibration, etc.), especially for the in-flight application where the reliability of ice protection must be ensured. Therefore, ice adhesion strength is no longer the sole criterion to evaluate the icephobic performance of a coating or a surface. It is a need to establish a more practical strategy for the design of icephobic coatings and surface. In this work, an energy saving strategy is proposed to assess the de-icing performance of the icephobic coating and surface when active heating is involved. The energy consumed for the de-icing operation assisted by the ice gravity is used as the key criterion for the overall performance of icephobic coating and surface. Successful validation has been achieved for evaluating the deicing performance of selected coatings and surfaces, which demonstrates an alternative strategy for the design and practical application of icephobic coatings and surfaces in ice protection.
\end{abstract}

Keywords: Energy saving, ice protection, de-icing, icephobic coatings, electro-thermal heating. 


\section{Introduction}

Aircraft is frequently exposed to cold environments and ice accumulation on aircraft surface may lead to catastrophic accidents, especially during aircraft landing, taking off and high-level cruise $[1,2]$. An effective solution of ice protection is a critical requirement in the aerospace industry for safety operation, fuel efficiency, and overall flight performance [3]. Current ice protection methods can be classified into two approaches: (i) active ice protection using mechanical, chemical, thermal or electrical techniques, etc. to remove the built-up ice from the component surface or to prohibit the ice formation at particular positions [4-7]; and (ii) passive ice protection which refers to the use of icephobic coatings or surfaces to delay and minimize the formation of ice, and lower the ice adhesion strength to facilitate the removal of ice naturally (e.g. by gravity and wind force, etc. [8, 9]. For the research and development (R\&D) of icephobic coatings, the current coating design target is mainly aiming to lower the ice adhesion strength between the ice and the coating surface [10]. Various icephobic coating system has been proposed based on this concept, including superhydrophobic surface [11-13], slippery liquid-infused porous surfaces [14, 15], and elastic coatings [16, 17], etc. Some typical evaluation methodologies for icephobicity have been reported, including both anti-icing and de-icing performance, like droplet icing test and ice adhesion test in cold environments [18-20].

However, as a passive ice protection approach, the use of icephobic coatings often has to be combined with an active ice protection solution in a critical condition, especially for the in-flight application where the reliability of ice protection must be ensured [21-23]. Applying additional heating for antiicing and de-icing is currently a major solution in the most civilian aircraft $[6,24]$, either by electrothermal heating or hot air bleeding technique. Therefore, ice adhesion strength is no longer the sole 
criterion to evaluate the icephobic performance of a coating and a surface. It is a need to establish a better strategy for the design of icephobic coating and surface, e.g. from the energy consumption and efficiency points, which would be in better compliance with the practical applications.

In this work, an energy saving strategy is proposed for the design of icephobic coatings and surface, and an electro-thermal heating approach assisted by the ice gravity is established to assess the deicing performance of the icephobic coatings and surface when heating is involved. The energy consumed for de-icing is used as the key criterion for the overall performance of icephobic coating and surface. Specimens with different surface roughness, coating thickness, conductivity, and hydrophobicity were examined using the electro-thermal de-icing testing in a cold environment. Successful validation has been clearly demonstrated in the evaluation of the de-icing performance of various surfaces, which provides an alternative strategy for the design and practical applications of icephobic coatings and surface for ice protection.

\section{Principle and methodologies}

\subsection{Energy criterion in electro-thermal de-icing evaluation}

De-icing by heating is normally an issue of heat conduction from a heating source beneath the concerned surface. To simplify the test, a one-dimensional multi-lamina heat conduction model was applied, and the schematic diagram of the test configuration is shown in Fig. 1, which consisted of a heating sheet (mat), an insulation sheet, an aluminium plate and a testing specimen attached with a cubic glaze ice. The heating sheet was used to create electrical heating and the insulation (thermal protective) layer on top was to minimise the energy loss from the opposite side of the testing specimen. 
A thermocouple was inserted between the insulation layer and the heating mat to monitor the temperature change. An aluminium plate was located below the heating sheet as a buffer, to provide constant heat transfer from the heating mat to the testing specimen. Thermal interface sheets with different thermal conductivies may be added separately to adjust the heat conduction. A glaze ice cube was frozen on the specimen surface in advance and faced down during the test. When enough heat flux had flowed to the testing specimen, the attached ice cube started to melt and weakened the ice adhesion, and finally dropped when its gravity force was higher than ice adhesion force. The overall time used for electro-thermal heating and the real-time temperature of the thermocouple were recorded until the falling of the ice cube. Then the energy consumption for removing the ice cube could be obtained, which varied with the different specimens and coatings.

\subsection{Materials}

Three types of thermal interface sheets were purchased from RS Components Ltd., UK, with thermal conductivities of 1,10 and $1600 \mathrm{~W} / \mathrm{m} \cdot \mathrm{K}$, respectively. A Kapton insulated flexible heating mat was supplied by OMEGA Engineering Ltd. UK. 1H, 1H, 2H, 2H-Perfluorooctyltriethoxysilane (POTS) were purchased from Sigma-Aldrich UK. Polydimethylsiloxane (PDMS) based R-1009 (RTV Silicone Dispersion Coating) resin was ordered from NuSil Technology LLC.

\subsection{Preparation of the testing specimens}

The details and preparation of specimens evaluated in the electro-thermal de-icing test were listed in Table 1. Pure aluminum plates were cut into $34 \mathrm{~mm} \times 34 \mathrm{~mm} \times 0.7 \mathrm{~mm}$ and used as testing specimens and coating substrates. The as-received Al plates without further surface treatment were assigned as 
smooth aluminum (SA). A batch of the Al specimens were sandblasting treated using Guyson Formula 1200 cabinet using $1 \mathrm{~mm}$ white alumina and named as rough aluminum (RA). To reduce the surface energy of the aluminium surface, $1 H, 1 H, 2 H, 2 H$-Perfluorooctyltriethoxysilane (POTS) selfassembled monolayer was deposited on SA and RA surface using chemical vapour deposition method in a sealed vessel with $0.3 \mathrm{ml}$ POTS at $180^{\circ} \mathrm{C}$ for 3 hours, as reported elsewhere [25]. SA and RA specimens modified with POTS were named as PSA and PRA, respectively.

To investigate the influence of heat conduction, thermal interface sheets with different thermal conductivities were assembled between the aluminium buffer plate and the testing specimen, as indicated in Fig. 1. For PDMS-coated specimens, SA and RA substrates were coated with PDMS R1009 using a KW-4A spin coater. The coater was set with an initial speed of 500 rpm for 9 seconds followed by $2,000 \mathrm{rpm}$ for 60 seconds. Then the coated specimen was put into to an oven at $65^{\circ} \mathrm{C}$ for 5 minutes to evaporate the solvent. The spin coating operation was repeated for a few cycles to obtain the desired coating thickness. Finally, the coatings were cured at $110^{\circ} \mathrm{C}$ for 8 hours, and then naturally cooled down to room temperature. By adjusting the operation cycles, PDMS coatings with thickness of 20, 43 and $77 \mu \mathrm{m}$ were produced, and assigned as PDMS-SA-1, PDMS-SA-2, and PDMS-SA-3, respectively. 
Table 1 List of specimens measured in the electro-thermal test

\begin{tabular}{ll}
\hline $\begin{array}{l}\text { Abbreviation } \\
\text { of specimens }\end{array}$ & \multicolumn{1}{c}{ Details of specimens } \\
\hline SA & Smooth aluminium plate (substrate) \\
RA & Rough aluminium plate (substrate) \\
PSA & Smooth aluminium plate coated with POTS monolayer \\
PRA & Rough aluminium plate coated with POTS monolayer \\
PSA-TC1 & PSA coupled with 1 $\mathrm{W} / \mathrm{m} \cdot \mathrm{K}$ thermal interface sheet (TC1) \\
PSA-TC10 & PSA coupled with 10 $\mathrm{W} / \mathrm{m} \cdot \mathrm{K}$ thermal interface sheet (TC10) \\
PSA-TC1600 & PSA coupled with $1600 \mathrm{~W} / \mathrm{m} \cdot \mathrm{K}$ thermal interface sheet (TC1600) \\
PDMS-SA & Smooth aluminium plate coated with PDMS \\
\hline
\end{tabular}

\subsection{Characterisation}

Hydrophobicity of the surfaces was characterized using an FTA200 contact angle goniometer (First Ten Angstroms) with pumping out rate of $1 \mu 1 /$ second. Both static water contact angles and water contact angle hysteresis of the specimens were obtained. The surface compositions and binding energy of the related elements were characterized by an X-ray photoelectron spectroscopy (XPS) (ESCALAB Mark II, VG Scientific, Waltham, MA, USA). And the results were further analysed using CasaXPS software. The surface roughness of specimens was evaluated by a Form 50 Talysurf machine, and each specimen was measured at 5 different areas. The average of surface roughness (Ra) of the specimen was then calculated. The thickness of the PDMS coatings was measured by a caliper at five points to study the influence of coating thickness on the de-icing performance.

\subsection{Electro-thermal Test}

Electro-thermal heating test was performed using a setup described in Section 2.1. The test was conducted in an environmental chamber with the temperature of $-5 \pm 0.2^{\circ} \mathrm{C}$. A precisely prepared glaze ice cube $(20 \mathrm{~mm} \times 20 \mathrm{~mm} \times 20 \mathrm{~mm})$ was attached to the specimen surface and faced down for the testing. When the environmental temperature was stabilized, electro-thermal heating was applied with 
a power of $1.35 \mathrm{~W}$ (input voltage $15 \mathrm{~V}$ ). The heating duration and the temperature change of the thermal couple were recorded, until the ice cube dropped from the specimen surface. The total energy consumption for each testing specimen would be calculated and used to evaluate the de-icing performance of the studied specimen surface.

\section{Results}

\subsection{Outputs of electro-thermal de-icing test}

There are two key experimental outputs from the electro-thermal heating test: melting time of ice cube prior to dropping, and the temperature of the heating source versus heating time. Fig. 2 shows the temperature changes of the heating sheet during the melting of PSA coupled with different thermal interfaces. The importance of heat conduction had been confirmed by the results. When the thermal interface with the highest thermal conductivity (e.g. $1600 \mathrm{~W} / \mathrm{m} \cdot \mathrm{K}$ ) was used, the shortest melting time of ice cube was observed, as heat could be more effectively conducted to the surface of the testing specimen which led to less melting time. The power output of the heating sheet was fixed at $1.35 \mathrm{~W}$, and the overall energy consumption in a test was directly proportional to the melting time of the ice cube attached on the testing specimen. It is interesting to find that the temperature of the heating source was lower at a given time when using a thermal interface with higher thermal conductivity, which was attributed to better heat conduction from the heating sheet to the ice cube.

\subsection{Electro-thermal test results of pure aluminium plates and POTS coated plates}

Table 2 summarises the surface features and electro-thermal testing results of the specimens, including surface roughness, surface wettability, melting time of ice cubes, and the energy 
consumption. From the energy saving point, the SA plates coated with POTS monolayer demonstrated the best performance for removing the ice cubes. However, once thermal interface sheet with low thermal conductivity (e.g. TC1) was used, a significant increase in energy consumption was clearly observed, confirming the dominating role of heat conduction in de-icing with heat.

Table 2 Testing results of energy consumption, surface wettability, and surface roughness

\begin{tabular}{lcccc}
\hline \multicolumn{1}{c}{ Type of specimens } & $\begin{array}{c}\text { Surface roughness } \\
\mathrm{Ra} / \mu \mathrm{m}\end{array}$ & $\begin{array}{c}\text { Water contact } \\
\text { angle, WCA }{ }^{\circ}\end{array}$ & Melting time /s & $\begin{array}{c}\text { Energy } \\
\text { consumption /J }\end{array}$ \\
\hline SA & $0.22 \pm 0.10$ & $74 \pm 0.9$ & $249 \pm 8$ & $336 \pm 11$ \\
RA & $0.68 \pm 0.10$ & $83 \pm 2.2$ & $284 \pm 10$ & $383 \pm 14$ \\
& & & & \\
PSA & $0.25 \pm 0.10$ & $102 \pm 1.8$ & $221 \pm 10$ & $298 \pm 14$ \\
& & & & \\
PRA & $0.64 \pm 0.10$ & $123 \pm 1.9$ & $248 \pm 7$ & $335 \pm 9$ \\
PSA with TC1 & $0.25 \pm 0.10$ & $102 \pm 1.8$ & $364 \pm 14$ & $491 \pm 19$ \\
PSA with TC10 & $0.25 \pm 0.10$ & $102 \pm 1.8$ & $355 \pm 13$ & $479 \pm 18$ \\
PSA with TC1600 & $0.25 \pm 0.10$ & $102 \pm 1.8$ & $233 \pm 5$ & $315 \pm 7$ \\
PDMS-1 & $0.07 \pm 0.05$ & $112 \pm 4.3$ & $231 \pm 6$ & $312 \pm 8$ \\
PDMS-2 & $0.08 \pm 0.05$ & $107 \pm 1.7$ & $242 \pm 9$ & $327 \pm 12$ \\
PDMS-3 & $0.11 \pm 0.05$ & $109 \pm 2.0$ & $256 \pm 8$ & $346 \pm 11$ \\
\hline
\end{tabular}

\subsection{Influence of surface roughness}

It is well believed that surface roughness will take a key role on icephobic performance [26]. Surface roughness data is given in Table 2 . There are two basic types of specimens for roughness study: the as-received SA plates and the sandblasted RA plates, which had the Ra values around $0.22 \mu \mathrm{m}$ and $0.68 \mu \mathrm{m}$, respectively. After coated with a POTS thin layer, the surface roughness is almost unchanged for both SA and RA substrates. De-icing energy used for these 4 typical specimens is compared in Fig. 3a. There is a clear trend on the influence of surface roughness: higher roughness leads to higher 
energy consumption, and this principle is applicable for both un-coated and POTS-coated plates. Fig. $3 \mathrm{~b}$ shows the temperature changes of heating sheet versus the heating time for the 4 typical specimens. Besides the difference in dropping time, it is interesting to find that there are also some variations on temperature changes. As described in Section 3.1, the variation of heating source temperature can be treated as an indicator of heat conduction during the test. The temperature curve of RA surface is above that of SA surface in Fig. 3b, it shows that RA specimens caused lower heat conduction in the test, which is not favorable from the energy efficiency point. A similar trend is also observed in the comparison of PSA and PRA results.

\subsection{Effects of surface wettability and surface energy}

Although there are some arguments on the relationship between the surface wettability and icephobicity, hydrophobicity is still a popular option for the development of icephobic coatings and surfaces. Some link between hydrophobicity and icephobicity has been reported [27-29], and water contact angle (WCA) and water contact angle hysteresis (CAH) are two the major parameters evaluating hydrophobic performance of coatings. Water contact angles of the different specimens are listed in Table 2. However, from the values of water contact angle, specimens with higher water contact angles do not automatically demonstrate better energy saving in the test. To further study the influence of surface wettability, water contact angle hysteresis of the specimens were also measured. Fig. 4 shows the correlations of melting time versus WCA (Fig.4a), and melting time versus CAH (Fig. 4b), in which both excludes the results of using the additional thermal interface sheets. Again, no direct advantage had been found with higher surface hydrophobicity and lower CAH from the energy consumption point. The influence of surface WCA and CAH on the energy used seems to be 
more randomly, indicating that neither surface WCA nor CAH did play a major role in the energy saving strategy.

The wettability of a solid surface is determined by its surface energy and surface morphology. In this study, the surface energy of the Al plate specimens was adjusted with the POTS thin layer without significant change on the surface morphology, as indicated by the roughness data in Table 2 . Due to the high content of C-F groups in POTS structure, the POTS layer would significantly reduce the surface energy [26], and it offered a kind of "non-stick" feature to the surface, which is also applicable to the attached ice. According to the energy consumption in Fig. 3a, it is quite encouraging that energy used for both smooth and rough specimens effectively decreased with the additional POTS layer: from $336 \mathrm{~J}$ and $383 \mathrm{~J}$ to $298 \mathrm{~J}$ and $335 \mathrm{~J}$, respectively. It is confirmed that the POTS layer is a benefit to the removal of the ice.

\subsection{Influence of PDMS coating and coating thickness}

As a typical hydrophobic polymer, PDMS is often considered as a candidate coating material for the icephobic application. From Table 2, it is found that when PDMS coating was applied on the SA plates, the overall roughness has a slight decrease, and the surface wetting behaviour changed from hydrophilic to hydrophobic, indicated by the increase of WCA from $74^{\circ}$ to around $110^{\circ}$. In the electrothermal test, the melting time of ice cubes and energy consumption for PDMS coated SA specimens was dependent on the coating thickness. With $20 \mu \mathrm{m}$ PDMS (PDMS-SA-1), shortened melting time and lowered energy consumption were clearly observed, compared with those of un-coated SA plates. When the PDMS thickness increased to $43 \mu \mathrm{m}$, the effectiveness in energy saving was reduced. While 
negative influence on energy saving was observed when the coating thickness became $77 \mu \mathrm{m}$. The surface roughness and surface energy of these PDMS coated SA specimen were quite similar, it is likely that differences in melting time and energy used were contributed to the heat conduction. The thermal conductivity of PDMS is around $0.15 \mathrm{~W} / \mathrm{m} \cdot \mathrm{K}$ [30], much lower than that of aluminum. A small increase in PDMS coating thickness showed a significant impact on heat conduction, which led to longer melting time and more energy consumption.

\section{Discussion}

In the proposed energy saving criterion, the ice cube under the gravity force is electrically heated. There are two major factors influencing the melting time and the energy consumption to remove the ice cube: (1) energy available to melt the ice layer at the interface, and (2) the ice adhesion which is being decreased due to the ice melting. Therefore, for icephobic coating and surface design, it is essential to work out the possible effects of the coatings and surfaces on heat conduction and the variations of ice adhesion, coating materials, surface morphology, and surface energy, etc.

\subsection{The role of heat conduction}

In the electro-thermal heating test, heat conduction is the major format of thermal transfer to melt the ice at the interface, and the system can be analyzed by a one-dimensional multi-lamina model, as shown in Fig. 5. In this model, $b_{n}$ is the layer thickness and $\delta_{n}$ is the thermal conductivity of each layer. The heat flux is considered as a one-dimensional flow from the high temperature side to the ice cube. In a given heating time, the energy available to melt the ice at the interface is proportional to the heat flow and the resistance to the heat conduction. $\sum b$ is the total thickness of the multi-lamina 
layer for the heat conduction, the heat resistances of each layer can be represented as a connection in series, consisting of a sum of reciprocal conductivities [31]. And the effective thermal conductivity $k_{\text {eff }}$ normal to the overall thickness could be calculated using Equation (1):

$$
k_{e f f}=\sum b / \sum_{n=1}^{\infty} \frac{b_{n}}{\delta}
$$

$\sum b / k_{e f f}$ in Equation (1) is referred to the overall heat transfer resistance per unit area, which is the value added together by the heat resistance of each individual layer. The layer with lower thermal conductivity and higher thickness would contribute more on the overall heat resistance, and thus more influence on the heat conduction in the system.

As material having high thermal resistance requires more energy to transfer certain heat and the slower transfer speed; Thermal resistance it can be calculated through equation (2):

$$
R=\frac{b}{\delta A}
$$

where $\mathrm{b}$ is the thickness of the layer; $\mathrm{A}$ is the conduction area and $\delta$ is the thermal conductivity. When thermal interface sheets were applied between the heating source and the ice cube, the additional thermal resistances directly impacted on the energy effectiveness on ice removal. The highest $k$ sheet (e.g. $1600 \mathrm{~W} / \mathrm{m} \cdot \mathrm{K}$ ) brought the lowest energy consumption in the ice removal, as observed in Fig. 2. As the addition of coating may greatly influence the heat transfer, for the design of icephobic coatings, low thermal resistance is an essential requirement.

Low thermal resistance means high thermal conductivity and low coating thickness. Heat conduction is actually sacrificed with the use of hydrophobic PDMS coating. As shown in Table 2, when a thin 
PDMS coating (e.g. $20 \mu \mathrm{m}$ ) was applied, the coated surface was beneficial from the addition of PDMS layer, as compared uncoated SA specimen. But the low thermal conductivity of PDMS makes the thermal conduction very sensitive to the coating thickness. With the increase of PDMS thickness, the energy consumption for de-icing significantly increased. With a thicker PDMS (e.g. $77 \mu \mathrm{m})$, the negative impact on the heat conduction dominated the thermal de-icing process and more energy had to be used, which was even higher than the uncoated SA reference surface. Therefore, to achieve the benefit of icephobic coating, the coating thickness is expected to be well controlled according to the energy saving strategy. From this perspective, the decrease of coating thickness would be beneficial for the de-icing performance, especially for the coating materials with low thermal conductivity.

\subsection{Influence of the Surface Roughness}

Surface roughness is another important factor which also plays a critical role in the de-icing performance. As the interface of the solid surface and the ice, surface roughness would have direct influences on both heat conduction and ice bonding. There is a clear comparison in Table 2 that after sandblasting, the rough specimens (e.g. RA) consumed more energy for de-icing, indicating that rougher surface is not favorable to remove the ice in the energy saving strategy. Similar trend was also observed with the POTS coated smooth and rough surface. With accretion of ice, rough surface would lead to high ice bonding strength $[19,32]$, as a result of possible mechanical interlocking and increase of surface contact area. Moreover, according to the temperature profile in Fig. 2, rough surface reduced heat conduction which also seemed be a major reason of high energy consumption. At the material-ice interface, air is possible to be trapped between the ice and solid surface during the accretion of ice, as illustrated in Fig. 6. Compared with the Al substrates and the coating materials, 
air has much lower thermal conductivity, e.g. $0.024 \mathrm{~W} / \mathrm{m} \cdot \mathrm{K}$ [31], contributing very little on heat conduction at the interface. The thermal contact resistance due to the non-flatness and roughness takes a huge effect on the heat transfer thought the material/ice interface as only limited contacting area is involved. The general thermal flux would be significantly reduced with a rough surface. Therefore, from the formation of mechanical interlocking and the increase of thermal contact resistance, rougher surface is not an advantage for the design of icephobic coatings and surface.

\subsection{Surface hydrophobicity}

Hydrophobic surface is currently still a popular research direction for icephobic application, and some arguments have been raised on the effectiveness of hydrophobicity on ice protection. The surface hydrophobicity, mainly reflected by WCA and $\mathrm{CAH}$, is determined by the surface energy of the coating materials, as well as the surface topography and roughness. It is a normal concept that a hierarchical surface structure in micro and nano scale would be a perfect configuration for hydrophobic surface [33], as the trapped air at the interface would be helpful for repelling the water droplets. This kind of hydrophobic surface would be helpful for anti-icing activity as water droplets would have less chance to stay on a hydrophobic surface. However, in the energy saving strategy, direct relationship is difficult to be established between the surface hydrophobicity and energy consumption. It is interesting that specimen PRA has the highest water contact angle among all the studied specimens, but the effectiveness in de-icing is not observed. The best energy saving is achieved with POTS coated SA surface. Higher water contact angle does not indicate greater likelihood of icephobicity. Similarly, CAH of the surface also has little influence on the de-icing performance in Fig. $4 \mathrm{~b}$, not as previously expected. The heat conduction and surface roughness seem 
to play more critical roles than the surface hydrophobicity. To pursue high surface hydrophobicity, high roughness or hierarchical roughness are often involved. However, as discussed in Section 5.2, higher roughness is clear a disadvantage in thermal de-icing, so that roughness induced hydrophobicity is not recommended for de-icing in the energy saving strategy.

From Fig.3a, based on the results from the similar surface roughness, it is very encouraged to find that all POTS coated Al substrates have better de-icing performance compared with their un-coated references. The low surface energy coatings would clearly offer lower ice bonding and thus contribute the energy saving in the test. Therefore, low-surface-energy induced hydrophobicity is still a solution for energy saving strategy.

To exclude the possible influence from surface elements, the surface compositions of the concerned surfaces (e.g. SA, RA, PSA, and PRA samples) were detected using XPS. The atomic percentages of the surface element contents of the four samples are given in Table 3. C and O elements on SA and RA samples mainly came from the adsorption of gases from the air (e.g. $\mathrm{O}_{2}$ and $\mathrm{CO}_{2}$, etc). While for PSA and PRA samples, after the deposition of POTS layer, their fluorine contents on the surface are very close to each other, $46.8 \%$ and $44.9 \%$, respectively. It confirms the validity of the study on the effects of surface wettability and roughness on energy consumption. 
Table 3 Comparison on atomic percentage of the surface elements of the sample surface

\begin{tabular}{cccccc}
\hline Atomic percentage & C (\%) & O (\%) & F (\%) & Al (\%) & Si (\%) \\
\hline SA & 46.8 & 28.7 & - & 24.5 & - \\
RA & 22.8 & 35.9 & - & 41.3 & - \\
PSA & 36.5 & 6.2 & 46.8 & - & 10.5 \\
PRA & 31.5 & 8.2 & 44.9 & - & 15.4 \\
\hline
\end{tabular}

Heating approach is a common solution of ice protection for aircraft, especially for in-flight de-icing methods, either by electro-thermal heating or hot air heating. Energy saving strategy brings an alternative evaluation approach for icephobic coatings and surfaces, aiming to reduce the overall energy consumption for ice protection. Low thermal resistance, low surface roughness and low surface energy would be the recommendation for such development.

\section{Conclusions}

An energy saving strategy was proposed for the R\&D of icephobic coatings and surfaces based on the strong dependence of thermal energy in ice protection. In this concept, a dedicated electro-thermal de-icing evaluation method was developed to verify the icephobic performance of typical specimens. The influence of heat conduction, roughness, and hydrophobicity of the coatings and surface had been verified.

Thermal conductivity of the coating materials, thickness of the coating, surface roughness, and surface energy are the major influencing factors to determine the thermal energy consumption. Low 
thermal resistance is required for the icephobic coatings and surfaces from the energy saving point, meaning high thermal conductivity and low coating thickness.

Although a rough surface would contribute to surface hydrophobicity for repelling water, due to the likelihood of forming mechanical interlocking and the increase of thermal contact resistance, rough surface is not an advantage for the design of icephobic coatings and surfaces in energy saving strategy. Coatings and surfaces with smooth surface finish would be recommended.

There is no direct relevancy between the surface hydrophobicity and the icephobicity from the energy saving point, including water contact angle and the contact angle hysteresis of the surface. Higher water contact angle induced by the surface roughness would bring negative impact on the de-icing. Low surface energy coatings would offer lower ice bonding and thus contribute to the energy saving ice protection. Therefore, low-surface-energy induced hydrophobicity is a still solution for energy saving strategy.

\section{Acknowledgment}

YZ would like to thank the support from Shanghai Nottingham Advance Academy (SNAA) program. This work was also supported in part by CleanSky 2 Joint Undertaking under the European Union's Horizon 2020 research and innovation programme under grant agreement No CS2-AIR-GAM-20142015-O1. Cf. Art.29.4 of [A2], as well as University of Nottingham Propulsion Futures Beacon funding. 


\section{References}

[1] I. Gultepe, R. Sharman, P.D. Williams, B. Zhou, G. Ellrod, P. Minnis, S. Trier, S. Griffin, S.S. Yum, B. Gharabaghi, A review of high impact weather for aviation meteorology, Pure and Applied Geophysics, 176 (2019) 1869-1921.

[2] I. Gultepe, A.J. Heymsfield, M. Gallagher, L. Ickes, D. Baumgardner, Ice fog: The current state of knowledge and future challenges, Meteorological Monographs, 58 (2017) 4.1-4.24.

[3] Y. Cao, W. Tan, Z. Wu, Aircraft icing: An ongoing threat to aviation safety, Aerospace Science and Technology, 75 (2018) 353-385.

[4] O. Parent, A. Ilinca, Anti-icing and de-icing techniques for wind turbines: Critical review, Cold regions science and technology, 65 (2011) 88-96.

[5] M. Pourbagian, W.G. Habashi, Aero-thermal optimization of in-flight electro-thermal ice protection systems in transient de-icing mode, International Journal of Heat and Fluid Flow, 54 (2015) 167-182.

[6] S.K. Thomas, R.P. Cassoni, C.D. MacArthur, Aircraft anti-icing and de-icing techniques and modeling, Journal of Aircraft, 33 (1996) 841-854.

[7] S. Venna, Y.-J. Lin, G. Botura, Piezoelectric transducer actuated leading edge de-icing with simultaneous shear and impulse forces, Journal of Aircraft, 44 (2007) 509-515.

[8] S. Tarquini, C. Antonini, A. Amirfazli, M. Marengo, J. Palacios, Investigation of ice shedding properties of superhydrophobic coatings on helicopter blades, Cold regions science and technology, 100 (2014) 5058.

[9] R. Flemming, Considerations on the Use of Hydrophobic, Superhydrophobic or Icephobic Coatings as a Part of the Aircraft Ice Protection System (2013-01-2108).

[10] A. Work, Y. Lian, A critical review of the measurement of ice adhesion to solid substrates, Progress in Aerospace Sciences, 98 (2018) 1-26.

[11] S. Farhadi, M. Farzaneh, S. Kulinich, Anti-icing performance of superhydrophobic surfaces, Applied Surface Science, 257 (2011) 6264-6269.

[12] C. Antonini, M. Innocenti, T. Horn, M. Marengo, A. Amirfazli, Understanding the effect of superhydrophobic coatings on energy reduction in anti-icing systems, Cold Regions Science and Technology, 67 (2011) 58-67.

[13] J. Liu, J. Wang, H. Memon, Y. Fu, T. Barman, K.-S. Choi, X. Hou, Hydrophobic/icephobic coatings based on thermal sprayed metallic layers with subsequent surface functionalization, Surface and Coatings Technology, 357 (2019) 267-272.

[14] P. Kim, T.-S. Wong, J. Alvarenga, M.J. Kreder, W.E. Adorno-Martinez, J. Aizenberg, Liquid-infused nanostructured surfaces with extreme anti-ice and anti-frost performance, ACS nano, 6 (2012) 65696577.

[15] S. Sunny, N. Vogel, C. Howell, T.L. Vu, J. Aizenberg, Lubricant-infused nanoparticulate coatings assembled by layer-by-layer deposition, Advanced Functional Materials, 24 (2014) 6658-6667. 
[16] K. Golovin, S.P. Kobaku, D.H. Lee, E.T. DiLoreto, J.M. Mabry, A. Tuteja, Designing durable icephobic surfaces, Science advances, 2 (2016) e1501496.

[17] C. Wang, T. Fuller, W. Zhang, K.J. Wynne, Thickness dependence of ice removal stress for a polydimethylsiloxane nanocomposite: Sylgard 184, Langmuir, 30 (2014) 12819-12826.

[18] D. Anderson, A. Reich, D. Anderson, A. Reich, Tests of the performance of coatings for low ice adhesion, in: 35th Aerospace Sciences Meeting and Exhibit, 1997, pp. 303.

[19] M. Zou, S. Beckford, R. Wei, C. Ellis, G. Hatton, M. Miller, Effects of surface roughness and energy on ice adhesion strength, Applied Surface Science, 257 (2011) 3786-3792.

[20] M. Wen, L. Wang, M. Zhang, L. Jiang, Y. Zheng, Antifogging and icing-delay properties of composite micro-and nanostructured surfaces, ACS applied materials \& interfaces, 6 (2014) 3963-3968.

[21] X. Huang, N. Tepylo, V. Pommier-Budinger, M. Budinger, E. Bonaccurso, P. Villedieu, L. Bennani, A survey of icephobic coatings and their potential use in a hybrid coating/active ice protection system for aerospace applications, Progress in Aerospace Sciences, 105 (2019) 74-97.

[22] T. Strobl, D.S. Thompson, M. Hornung, Feasibility Study of a Hybrid Ice Protection System Based on Passive Removal of Residual Ice, in: 53rd AIAA Aerospace Sciences Meeting, 2015, pp. 0032.

[23] Z. Wang, Recent progress on ultrasonic de-icing technique used for wind power generation, highvoltage transmission line and aircraft, Energy and Buildings, 140 (2017) 42-49.

[24] M. Mohseni, A. Amirfazli, A novel electro-thermal anti-icing system for fiber-reinforced polymer composite airfoils, Cold Regions Science and Technology, 87 (2013) 47-58.

[25] J. Liu, Z. Janjua, M. Roe, F. Xu, B. Turnbull, K.-S. Choi, X. Hou, Super-hydrophobic/icephobic coatings based on silica nanoparticles modified by self-assembled monolayers, Nanomaterials, 6 (2016) 232.

[26] J. Liu, J. Wang, L. Mazzola, H. Memon, T. Barman, B. Turnbull, G. Mingione, K.-S. Choi, X. Hou, Development and evaluation of poly (dimethylsiloxane) based composite coatings for icephobic applications, Surface and Coatings Technology, 349 (2018) 980-985.

[27] S. Kulinich, S. Farhadi, K. Nose, X. Du, Superhydrophobic surfaces: are they really ice-repellent?, Langmuir, 27 (2010) 25-29.

[28] L. Mishchenko, B. Hatton, V. Bahadur, J.A. Taylor, T. Krupenkin, J. Aizenberg, Design of ice-free nanostructured surfaces based on repulsion of impacting water droplets, ACS nano, 4 (2010) 7699-7707.

[29] S. Jung, M. Dorrestijn, D. Raps, A. Das, C.M. Megaridis, D. Poulikakos, Are superhydrophobic surfaces best for icephobicity? Langmuir, 27 (2011) 3059-3066.

[30] J. Mark, Polymer data handbook Oxford Univ, Press New York, NY p, 363 (1999).

[31] J.P. Holman, Heat Transfer, McGraw Hill Higher Education, 2010.

[32] Q. Fu, X. Wu, D. Kumar, J.W. Ho, P.D. Kanhere, N. Srikanth, E. Liu, P. Wilson, Z. Chen, Development of sol-gel icephobic coatings: effect of surface roughness and surface energy, ACS applied materials \& interfaces, 6 (2014) 20685-20692.

[33] L. Feng, S. Li, Y. Li, H. Li, L. Zhang, J. Zhai, Y. Song, B. Liu, L. Jiang, D. Zhu, Super-hydrophobic surfaces: from natural to artificial, Advanced materials, 14 (2002) 1857-1860. 


\section{List of Figure captions}

Fig. 1 Schematic diagram of the electro-thermal de-icing test under the ice gravity.

Fig. 2 Changes of temperature at heating source during the ice melting of PSA coupled with different thermal interfaces.

Fig. 3 Influence of POTS layer on aluminum plate in electro-thermal test: (a) energy consumption, and (b) temperature change at heating source.

Fig.4 The correlation between (a) water contact angle and melting time; (b) water contact angle hysteresis and melting time.

Fig. 5 Model schematic of thermal conductivity of multilayer layers.

Fig. 6 Schematic of sample surface with high roughness.

\section{List of Table captions}

Table 1 List of specimens measured in the electro-thermal test.

Table 2 Testing results of energy consumption, surface wettability, and surface roughness.

Table 3 Comparison on atomic percentage of the surface elements of the sample surface 


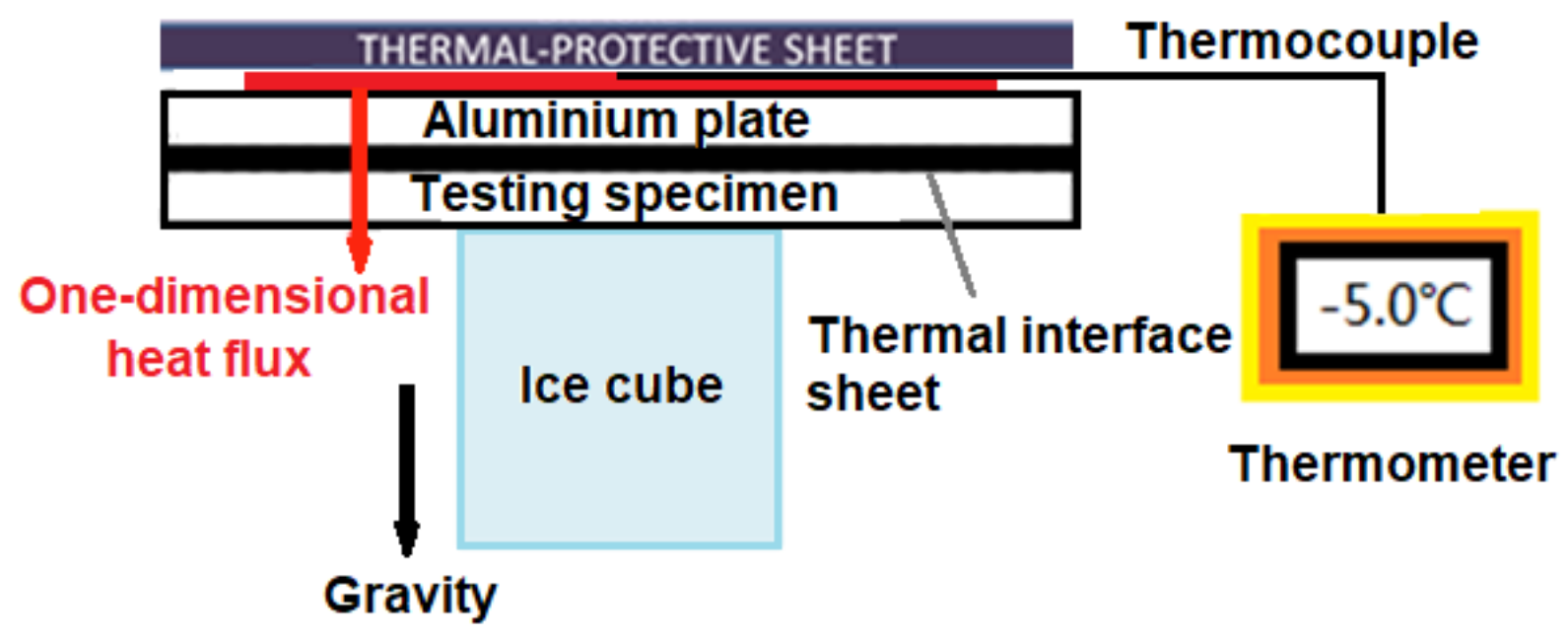

Fig. 1 Schematic diagram of the electro-thermal de-icing test under the ice gravity. 


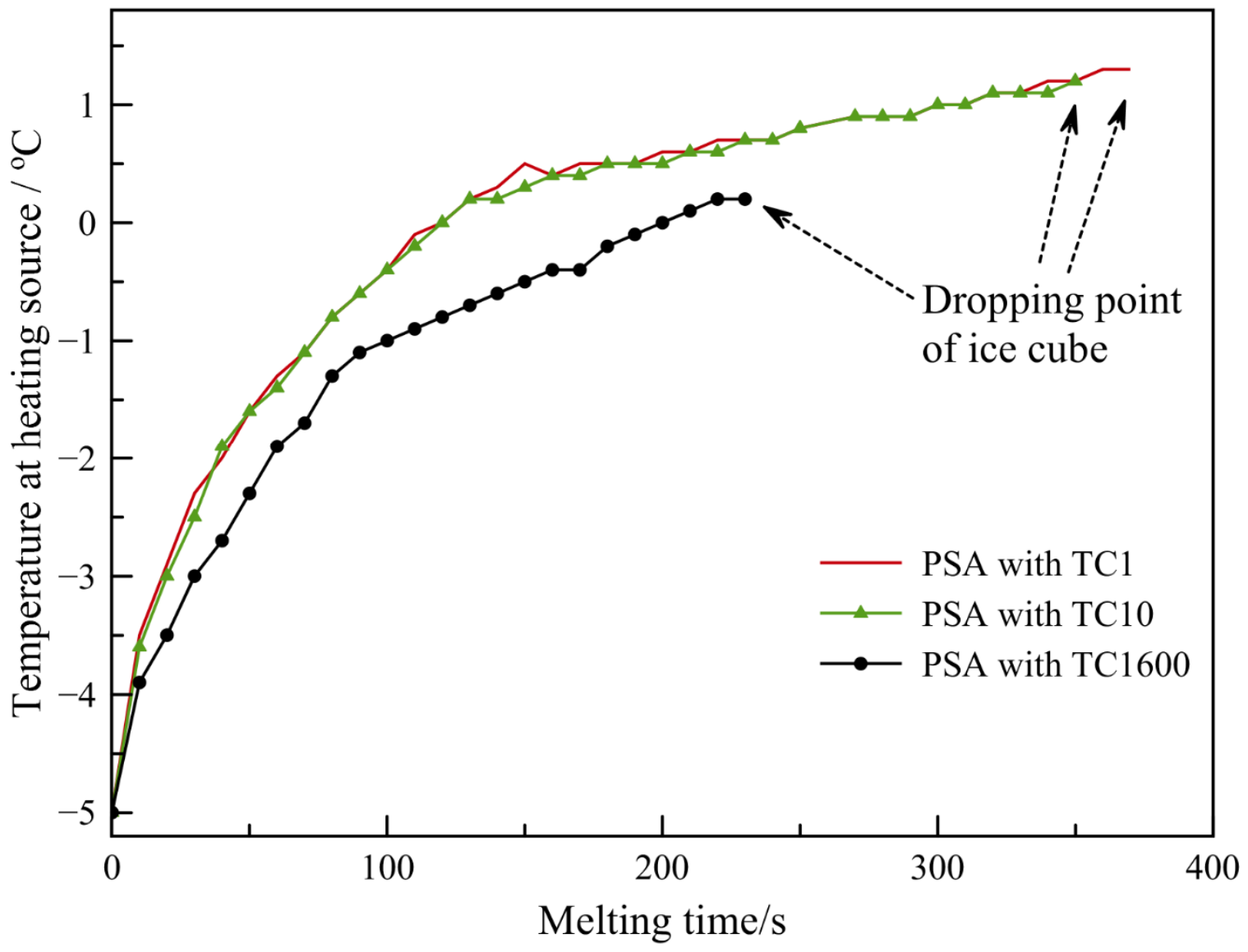

Fig. 2 Changes of temperature at heating source during the ice melting of PSA coupled with different thermal interfaces. 


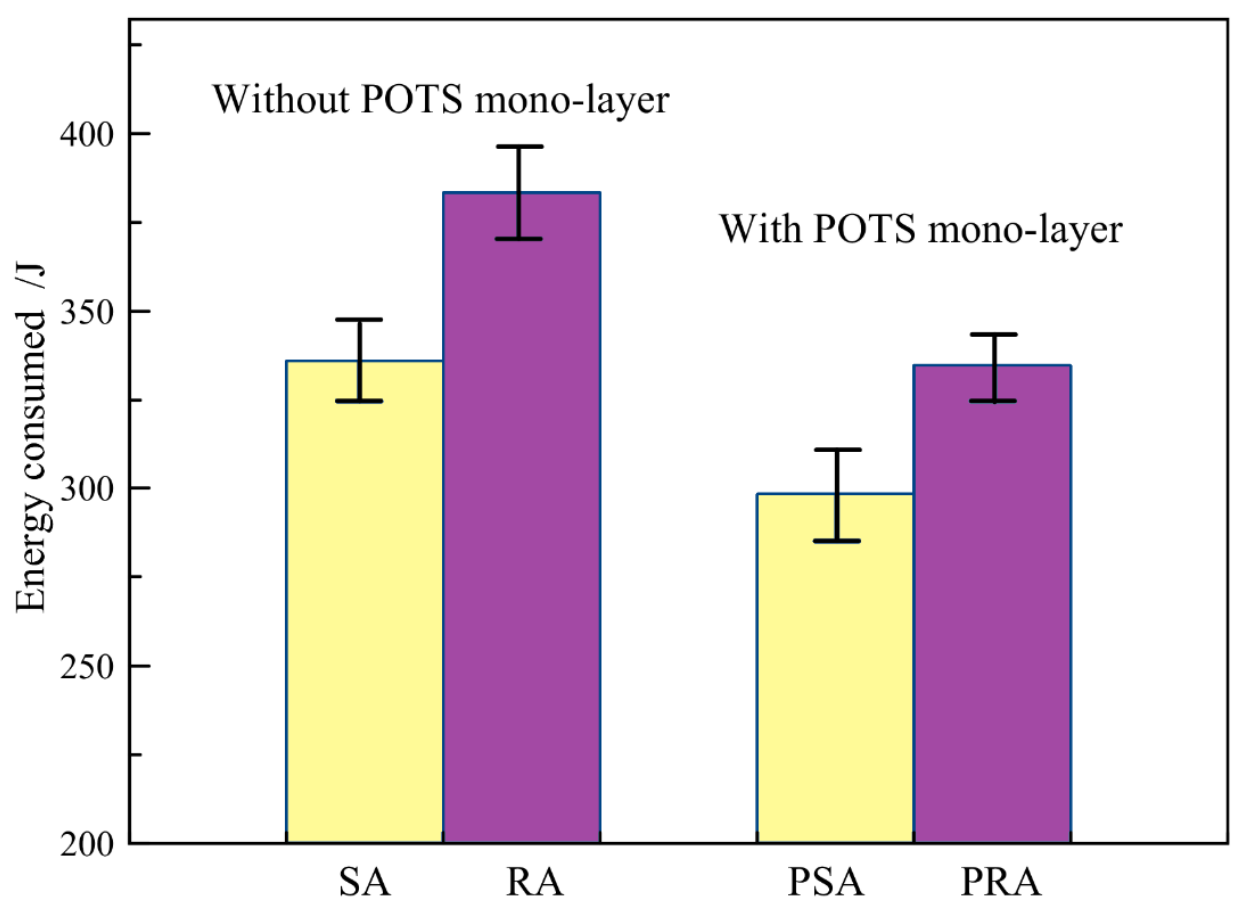

(a)

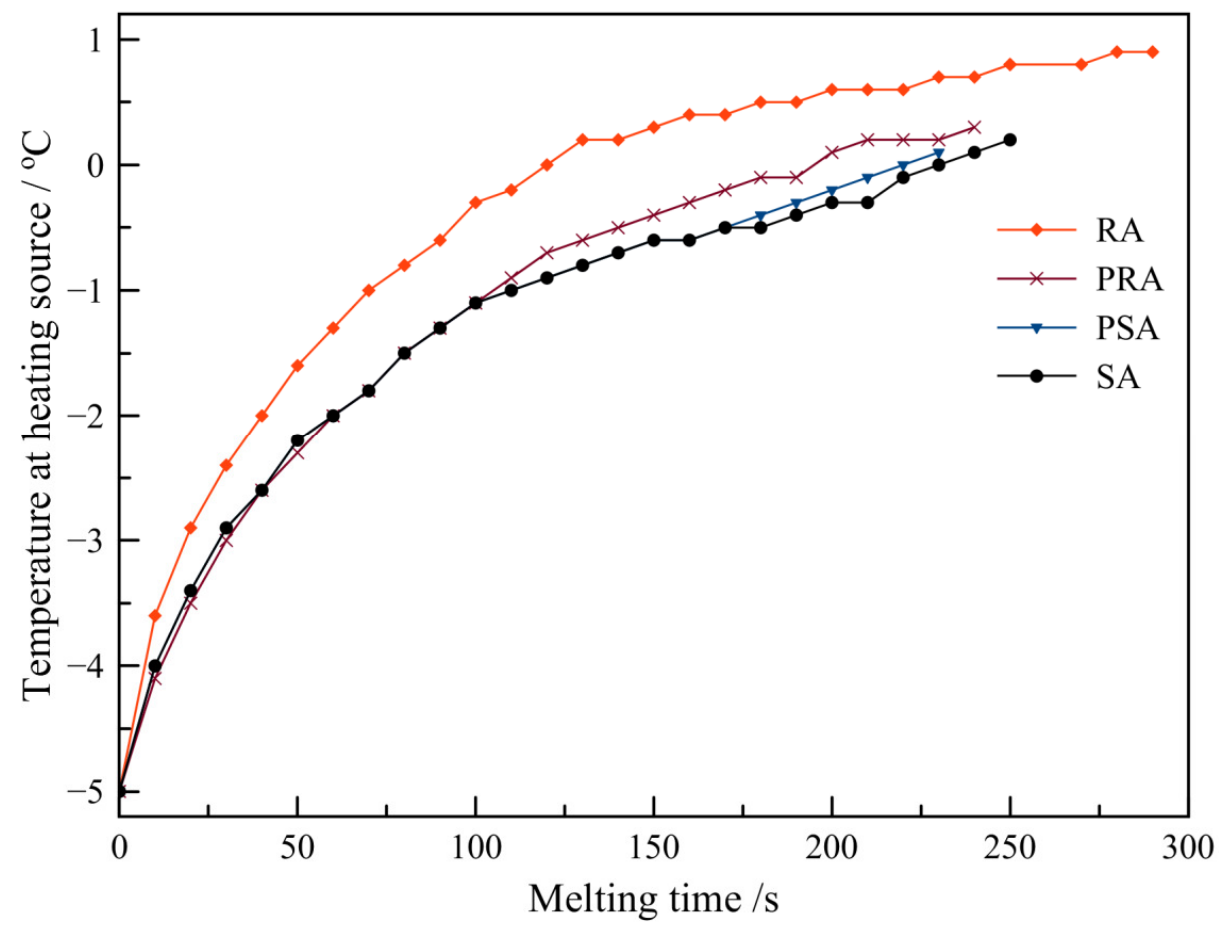

(b)

Fig. 3 Influence of POTS layer on aluminum plate in electro-thermal test: (a) energy consumption, and (b) temperature change at heating source. 


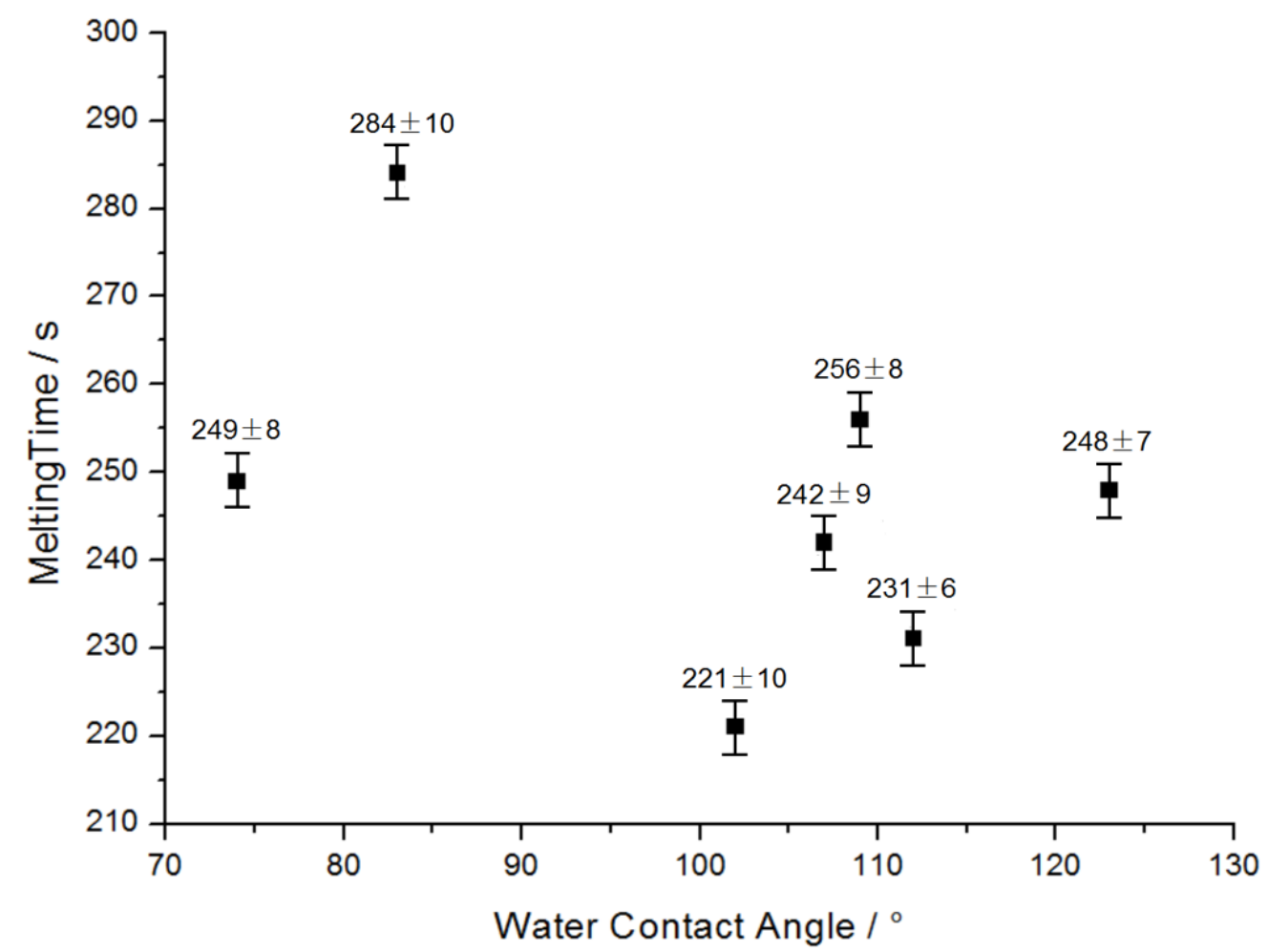

(a)

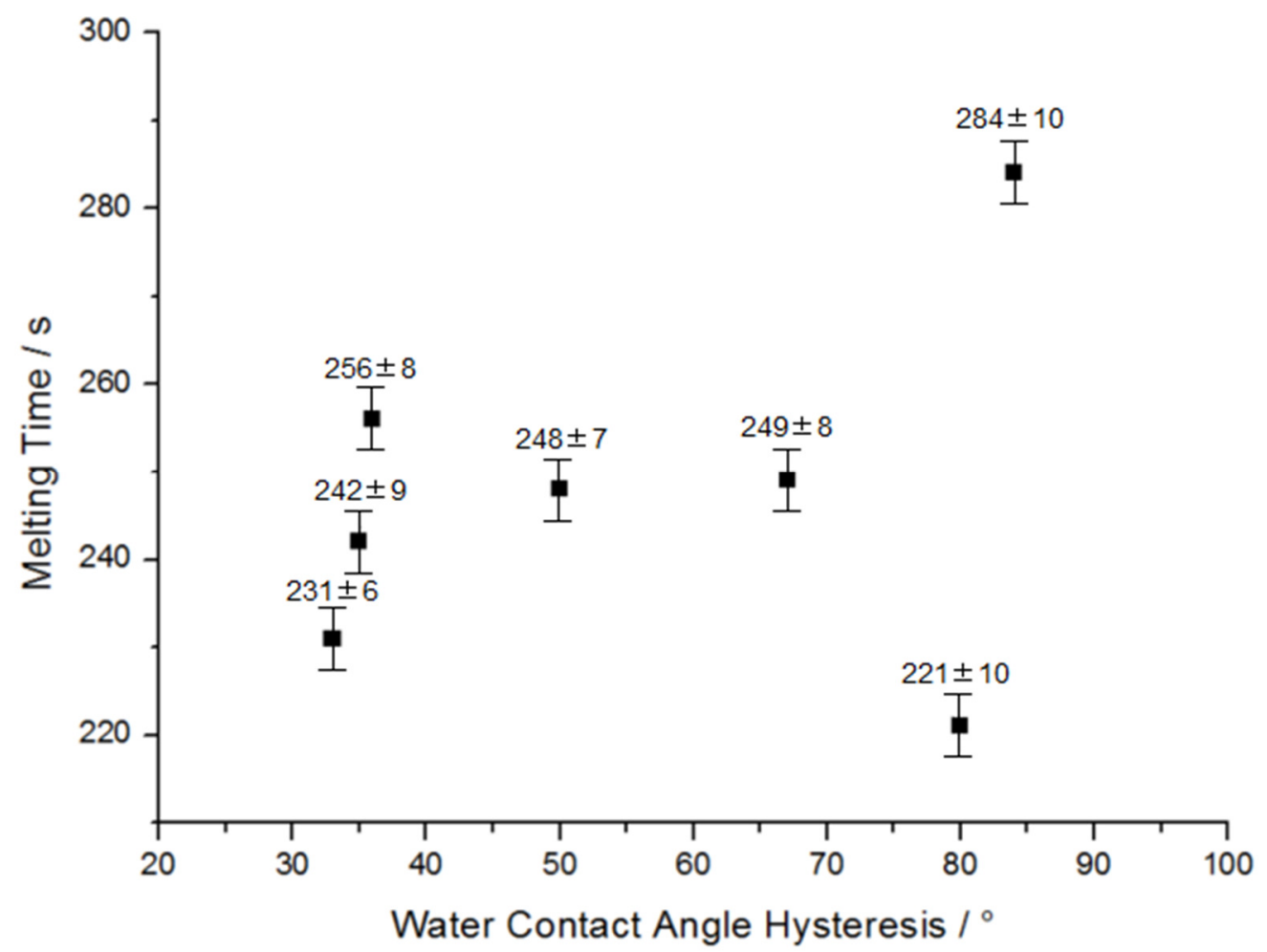

(b)

Fig.4 The correlation between (a) water contact angle and melting time; (b) water contact angle hysteresis and melting time 


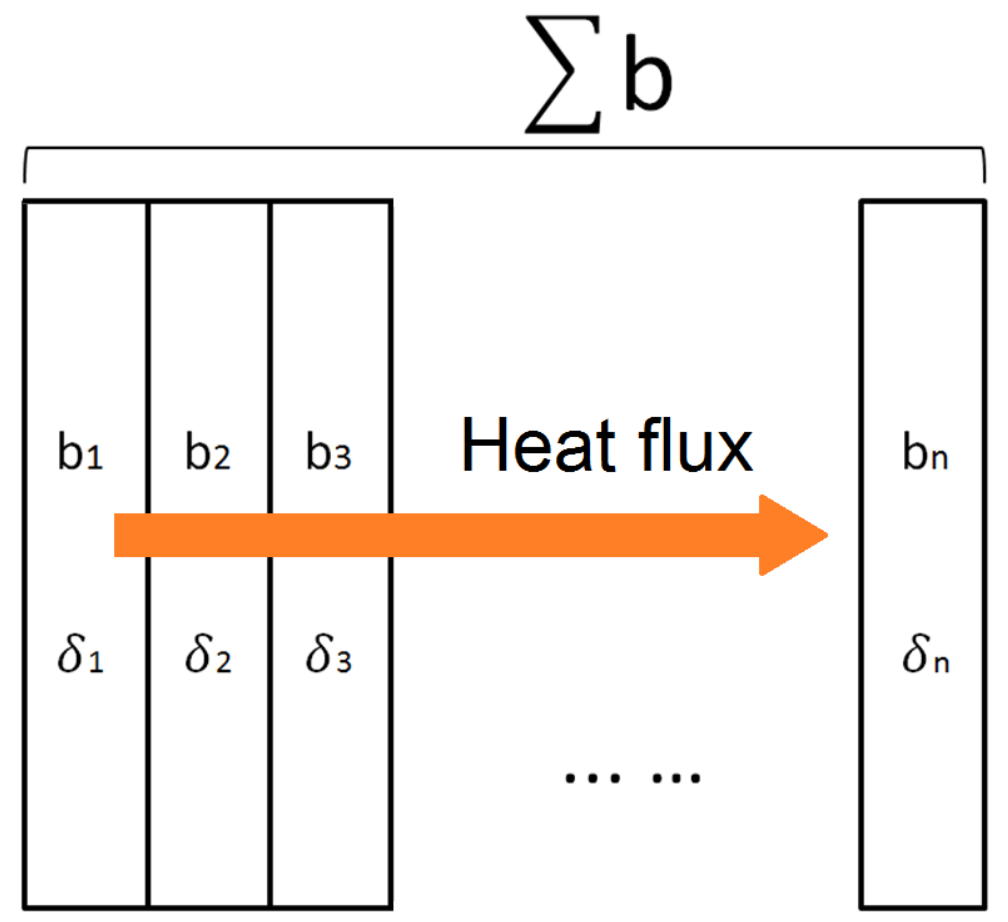

Fig. 5 Model schematic of thermal conductivity of multilayer layers 


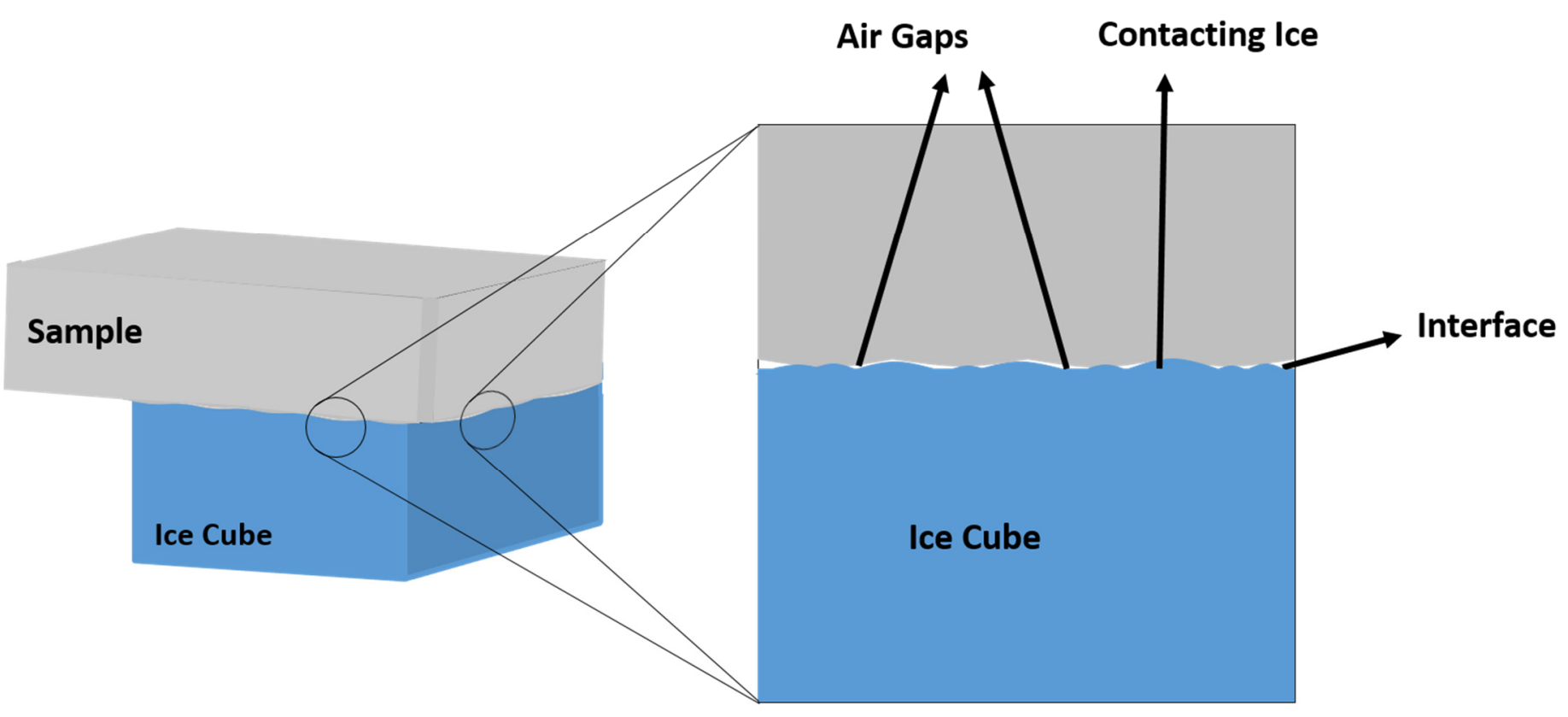

Fig. 6 Schematic of sample surface with high roughness 